\title{
Magnetization processes in rectangular versus rhombic planar superlattices of magnetic bars
}

\author{
Y. G. Pogorelov, ${ }^{1}$ G. N. Kakazei, ${ }^{1,2}$ J. M. Teixeira, ${ }^{1}$ A. Hierro-Rodriguez, ${ }^{3}$ F. Valdés-Bango, ${ }^{3}$ M. Vélez, ${ }^{3}$ J. M. Alameda, ${ }^{3}$ \\ J. I. Martín, ${ }^{3}$ J. Ventura, ${ }^{1}$ and J. B. Sousa ${ }^{1}$ \\ ${ }^{1}$ IFIMUP-IN (Institute for Nanoscience and Nanotechnology) and Departamento de Física, Universidade do Porto, \\ Rua Campo Alegre 687, 4169-007 Porto, Portugal \\ ${ }^{2}$ Institute of Magnetism, NAS of Ukraine, 36b Vernadskogo Blvd, 03142 Kiev, Ukraine \\ ${ }^{3}$ Departamento de Física, Universidad de Oviedo - CINN, c/Calvo Sotelo s/n, 33007 Oviedo, Spain \\ (Received 18 May 2011; revised manuscript received 14 July 2011; published 10 August 2011)
}

\begin{abstract}
Rectangular and rhombic patterned superlattices of magnetic bar elements have been experimentally studied and theoretically modeled in order to analyze the role of the array geometry in their magnetization reversal and coercivity. The results show that a dominating part of the coercive field $(\approx 250 \mathrm{Oe})$ is due to the reversal processes within a single bar element (independent of the array geometry) which is well described by the standard micromagnetic calculation. Otherwise, a smaller $(\approx 60 \mathrm{Oe})$ but significant difference between the magnetization loops in the two geometries is related to the magnetostatic coupling effects between the bars and it is reasonably accounted for with a simple model of Coulomb-like interaction between terminal magnetic charges. The possibility to use this geometry effect to control the performance of artificial magnetic media is discussed.
\end{abstract}

DOI: 10.1103/PhysRevB.84.052402

PACS number(s): 75.60.Ej, 75.60.Jk, 75.70.Kw, 75.75.Cd

Systems of magnetic micro- and nanoelements with dipolar or exchange coupling are interesting both for fundamental physics and for practical applications (e.g., for magnetic recording media). ${ }^{1-9}$ Fundamental interest is motivated by the possibility to controllably form nontrivial collective magnetic states $^{10}$ and manipulate phase transitions ${ }^{11,12}$ between them, controlled either by the material and shape parameters of nanoelements and by the geometric parameters of artificial superstructures. Such superstructures for prospective applications in spintronics and nanotechnologies are mainly studied in the simplest geometry of a square superlattice, having high enough symmetry ${ }^{13-15}$ which can hide however certain important manifestations of magnetic interactions.

To check the importance of the lattice geometry for the magnetic ground state and dynamical properties, we have studied magnetic properties of two artificial lattices made from Cobalt microbar elements and having rectangular (rec) and rhombic (rho) geometry. They were fabricated using the electron-beam lithography Raith system and liftoff techniques. The net magnetization $M$ was studied by Magneto-Optical Transverse Kerr effect. ${ }^{16}$ In this system, a laser beam is focused on a $300 \mu \mathrm{m}$ spot over the sample, sensing the average magnetic response of the whole array to the external magnetic field $H$ applied along the $x$-direction. The magnetic domain structures were probed by Nanotec Atomic Force Microscope with Nanosensors PPP-MFMR magnetic cantilevers in the magnetic force microscopy (MFM) measuring mode under applied in-plane magnetic field up to 950 Oe. Scanning electron microscopy images show that the fabricated Co elements are of $a=1.5 \mu \mathrm{m}$ and $b=0.3 \mu \mathrm{m}$ size (along the $x$ and $y$ Cartesian axes, respectively) at $0.3 \mu \mathrm{m}$ separation between them (Fig. 1), while their thickness was defined in the deposition process as $c=0.04 \mu \mathrm{m}$. The rec-and rho-superlattice geometries are coherent along all the patterned $200 \times 200 \mu \mathrm{m}$ square arrays.

The observed magnetization loops (Fig. 2), at reversing $H$ from its maximum value downward to negative values, maintain the initial saturation level $M \approx M_{S}$ until a sudden drop at a nucleation field $-H_{n}$, then passing $M=0$ at a coercive field $-H_{c}$ and finally reaching the inverted saturation $-M_{s}$ at the related field $-H_{s}$. The respective stages at reversing from $-H_{s}$ upward occur at positive $H_{d}, H_{c}, H_{s}$ values. Notably, all these characteristic fields in the rec-geometry: $H_{n} \approx 110 \mathrm{Oe}, H_{c} \approx 203 \mathrm{Oe}$, and $H_{s} \approx 331 \mathrm{Oe}$ are somewhat reduced compared to those in the rho-geometry: $\approx 186,272$, and $543 \mathrm{Oe}$, respectively. The slope $|d M / d H|$ is almost identical for the two geometries at the nucleation stage but becomes sizeably slower for the rho-geometry at the inversion stage. These geometry effects are undoubtedly beyond the experimental errors and so are due to magnetic interactions between the bar elements (see below).

A deeper insight on the underlying physics is obtained from the MFM data at sweeping the applied field from $H_{s}$ to $-H_{s}$ for each geometry. Figure 3 shows that the magnetization reversal mostly occurs through formation of specific linear domains (LD) along the $x$-direction. In the rec-case, these domains show more tendency to intercalation, that can be seen as "linear antiferromagnetism" (LAFM), while in the rho-case they more tend to merging or to ferromagnetism (FM), especially in the inversion stage as indicated by arrows in Figs. 3(c) and 3(g).

Discussing the magnetic states of the overall system, it is reasonable to distinguish between the two main tasks: first, the geometry independent hysteresis defined by reversal processes in an isolated element and, second, the geometry dependent magnetostatic interactions between elements, sensitive to the angles between their magnetizations and relative position vectors.

The reversal processes in an individual element can be suitably described within the common framework of micromagnetic OOMMF routine,${ }^{17}$ tracing the steps from the initial fully saturated state, through formation and propagation of certain vortex and domain structures within the bar, until reaching its full inverse saturation. The exact shape of fabricated elements and standard parameters of magnetization $M_{s} \approx$ $1400 \mathrm{emu} / \mathrm{cm}^{3}$, exchange constant $A \approx 3 \times 10^{-6} \mathrm{erg} / \mathrm{cm}$, and uniaxial anisotropy constant $K_{u} \approx 4 \times 10^{4} \mathrm{erg} / \mathrm{cm}^{3}$ were 


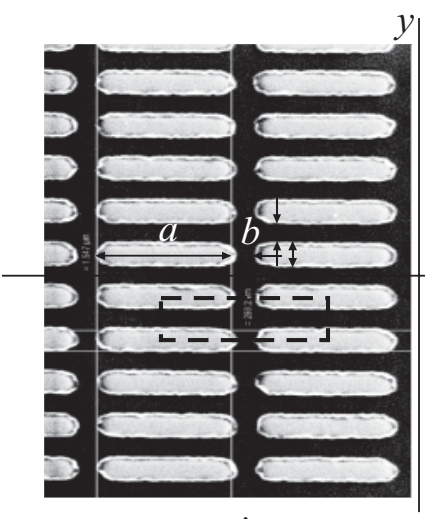

$i$

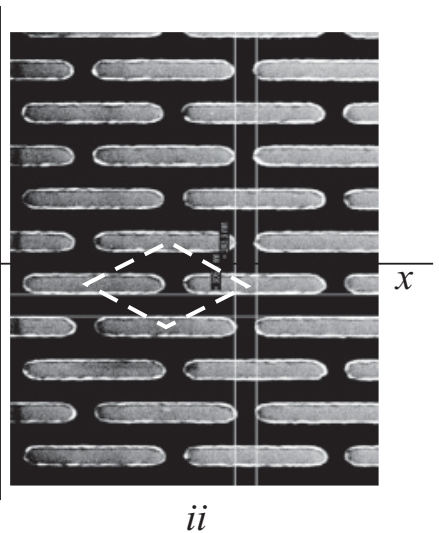

$i i$
FIG. 1. Bar elements of $a$ length, $b$ width, and $b$ separation, forming two types of superlattices: (i) rectangular (rec) and (ii) rhombic $(r h o)$. Dashed lines show the respective unit cells.

used. The resulting hysteresis loop shown in Fig. 2 is almost rectangular with the (micromagnetically defined) coercivity of $H_{m-m} \approx 250 \mathrm{Oe}$, close to the average value for two geometries in our samples.

The second task is about the correlations between completed reversals in neighbor elements, leading to formation of LD structures. It is based on the model magnetic energy of an array of fully saturated bar elements with Zeemann and magnetostatic interaction terms:

$$
E=m H \sum_{\mathbf{n}} \sigma_{\mathbf{n}}+\frac{\omega}{2} \sum_{\mathbf{n}^{\prime} \neq \mathbf{n}} \sigma_{\mathbf{n}^{\prime}} \sigma_{\mathbf{n}^{\prime}} U\left(\mathbf{n}-\mathbf{n}^{\prime}\right) .
$$

Here $m=M_{s} a b c$ is the saturated magnetic moment of a bar element, $\omega=m^{2} /\left(a b^{2}\right)$ is the interaction energy scale, and the Ising-like variable $\sigma_{\mathbf{n}}= \pm 1$ indicates the $x$-orientation of the

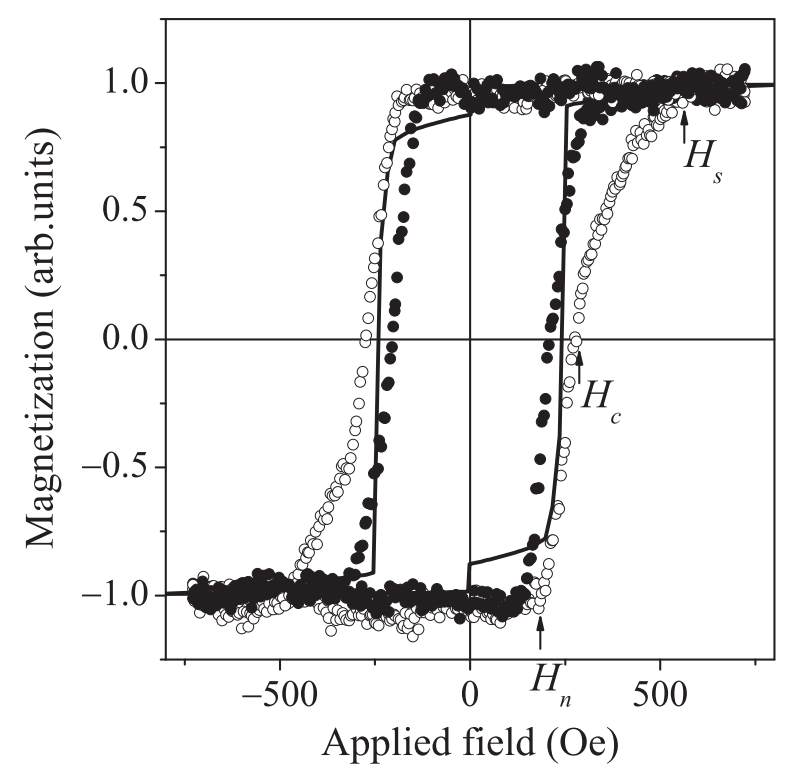

FIG. 2. Magnetization loops for rec (solid circles) and rho (open circles) structures. Solid line indicates the calculated hysteresis loop for an isolated bar element by the OOMMF routine. Arrows indicate the characteristic field values for upward reversal process in the rhogeometry. bar moment centered at the point $\mathbf{n}=(x, y)$ (see Fig. 1). The expression for $U(x, y)$ depends on specific shape of bars, and for $a \times b$ rectangles of negligible thickness, it is analytic:

$$
U(x, y)=2 F(x, y)-F(x+a, y)-F(x-a, y),
$$

where

$$
F(x, y)=\left[f\left(\frac{y+b}{x}\right)+f\left(\frac{y-b}{x}\right)-2 f\left(\frac{y}{x}\right)\right] \frac{x}{a}
$$

with $f(z)=z \operatorname{arcsinh} z-\sqrt{1+z^{2}}$ is the interaction between two lines of magnetic charges on bar $x$-faces (seen as dark and bright spots in the MFM images, Fig. 3) at separation $(x, y)$ between their midpoints. This $F(x, y)$ tends to the Coulomb $1 / r$ law, so $U(x, y)$ to the dipole-dipole $1 / r^{3}$ law, at long distances, $r=\sqrt{x^{2}+y^{2}} \gg a, b$, but deviates from it for close neighbors. The strongest interaction between the closest neighbors in the same row, $U(a+b, 0)$, results in the strongest FM correlation along each $x$-line and defines LD as the most robust structure in all transient states for both geometries of the array. But the lattice geometry is important for weaker correlations between LDs and so for collective magnetic behavior in each case.

An evident geometry sensitive value is the difference $\Delta \varepsilon_{\alpha}$ $(\alpha=r e c, r h o)$ of energies per element between the FM and LAFM states (for $H=0$ ). Its values calculated from Eqs. (1) and (2), $\Delta \varepsilon_{r h o} \approx-0.103 \omega$ and $\Delta \varepsilon_{r e c} \approx 0.238 \omega$, indicate FM ground state in the rho-case but LAFM in the rec-lattice. Notice that the "array transition temperatures" $T_{\alpha} \sim\left|\Delta \varepsilon_{\alpha}\right| / k_{\mathrm{B}}$ can be as huge as $\sim 10^{6} \mathrm{~K}$, making the FM ground state in the rho-case quite stable thermally, up to the Curie point $T_{c}$ of bar material (though easily reoriented by the applied field). But in the rec-case, the field-induced metastable FM state can stay "frozen" at all $T<T_{c}$, preventing to reach the true LAMF ground state.

As seen from the above MFM data (Fig. 3), the magnetization reversal in the considered arrays occurs through propagation of LDs, and the next important issue are the conditions for their stability upon their returning to the initial (noninverted) magnetic configuration. This stability can be characterized by the corresponding energy differences for a FM array in the presence of LD from $L$ elements and without it:

$$
\begin{aligned}
\delta \varepsilon_{r e c}= & -2 \sum_{i=1}^{L}\left[\sum_{n=L+1}^{\infty} u_{n-i, 0}+2 \sum_{n=1}^{\infty} \sum_{m=1}^{\infty} u_{n-i, m}\right], \\
\delta \varepsilon_{r h o}= & -2 \sum_{i=1}^{L}\left\{\sum_{n=L+1}^{\infty} u_{n-i, 0}\right. \\
& \left.+2 \sum_{n=1}^{\infty}\left[\sum_{m=1}^{\infty} u_{n-i, 2 m}+\sum_{m=1}^{\infty} u_{n-i+\frac{1}{2}, 2 m-1}\right]\right\},
\end{aligned}
$$

where $u_{n, m}=U[(a+b) n, 2 b m]$. These quantities calculated in function of $L$ (Fig. 4) rapidly attain linear asymptotics: $\delta \varepsilon_{\alpha} \rightarrow \delta_{\alpha}+L m h_{\alpha}$, with the characteristic field values $h_{r e c} \approx$ -9.7 Oe and $h_{r h o} \approx 4.1 \mathrm{Oe}$, so the (necessary) stability condition for each geometry, $H>h_{\alpha}$, is certainly assured for $H$ within the experimental range of magnetic reversal in Fig. 2. 

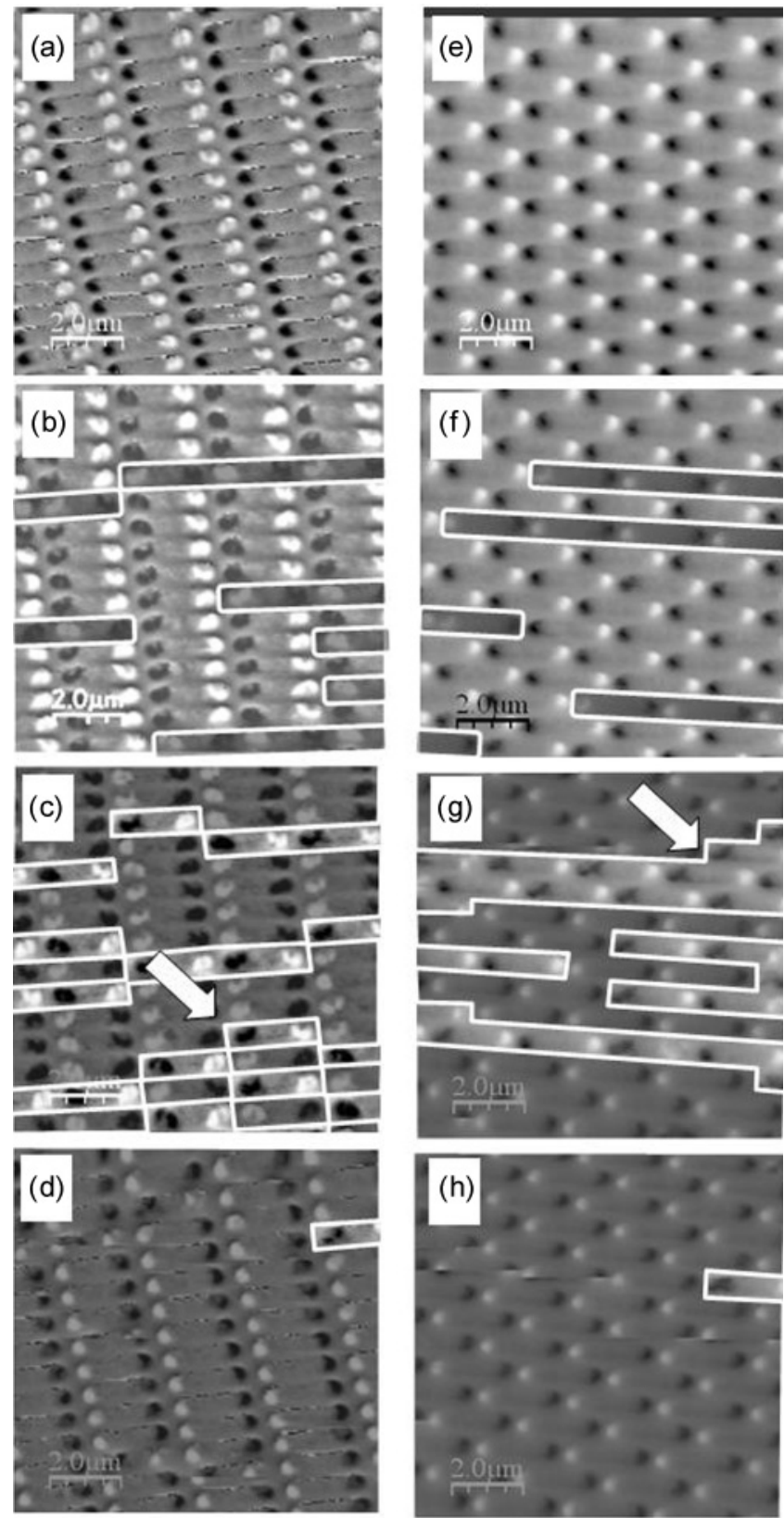

FIG. 3. Remagnetization processes at sweeping from positive to negative applied field for rec- (a)-(d) and rho- (e)-(h) superlattice geometries. Inverted areas in each case are outlined and shadowed, showing linear domains tendency to intercalation or "linear antiferromagnetism" in the rec-case and to merging or ferromagnetism in the rho-case (indicated by the arrows in panels $\mathrm{c}$ and $\mathrm{g}$ ).

Once LD stability is assured, the particular steps of its formation can be seen as a sequence of reversals in single elements along the LD line, under local fields acting on them. These fields result from the uniform external field and magnetostatic interactions of a given bar element with its neighbors. The reversal should be easiest in an element next to the "head" of an extending LD (Fig. 5), since its strongest interactions with neighbors in the same row are canceled. The reversal conditions for this element are alike those for

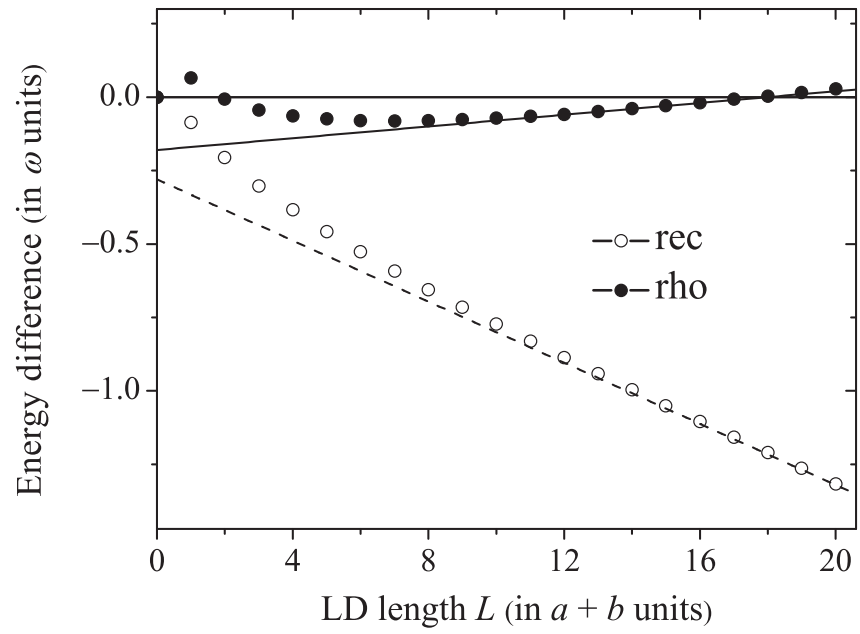

FIG. 4. Energy differences, $\delta \varepsilon_{\alpha}$, for an FM array with and without an LD vs its length $L$, and their linear asymptotics for $L \gg 1$. Open symbols and dashed line are for $\alpha=r e c$ while solid symbols and line are for $\alpha=r h o$.

an isolated bar, except for a relatively weak contribution to the reversal energy by interactions with neighbors in other rows:

$$
\begin{aligned}
\zeta_{\text {rec }} & =-2 \omega \sum_{n=-\infty}^{\infty} \sum_{m=1}^{\infty} u_{n, m}, \\
\zeta_{\text {rho }} & =-2 \omega \sum_{n=-\infty}^{\infty} \sum_{m=1}^{\infty} u_{n+\frac{1}{2}, m} .
\end{aligned}
$$

The calculated values: $\zeta_{\text {rec }} \approx-0.257 \omega$ and $\zeta_{\text {rho }} \approx 0.11 \omega$, define the respective contributions to the nucleation field $\delta H_{r e c}=\zeta_{r e c} / m \approx-48$ Oe and $\delta H_{r h o}=\zeta_{r h o} / m \approx 21$ Oe. Evidently, these $\delta H_{\alpha}$ values turn to be more relevant for the array reversal than the above considered $h_{\alpha}$.

Now, comparing all the contributions to the magnetic reversal, we conclude that the dominating one is the single-element $H_{m-m}$ value, while the difference between the geometry sensitive terms $\delta H_{r h o}-\delta H_{r e c} \approx 69$ Oe well agrees with the observed rec-rho difference of $H_{d}$ values.

At further advance of reversal, the slope $|d M / d H|$ is mainly controlled by the interactions between close LDs. In the adopted model, they are repulsive for $r e c$ - but attractive for rho-geometry as follows from the corresponding calculations based on Eqs. (1) and (2): $u_{r e c} \approx 0.114 \omega$ and $u_{r h o} \approx-0.055 \omega$ (per element). This can explain the observed tendency to LD merging and the slower approach to inverted saturation in the rho-case [by a higher resistance to reversal of the "frontier"

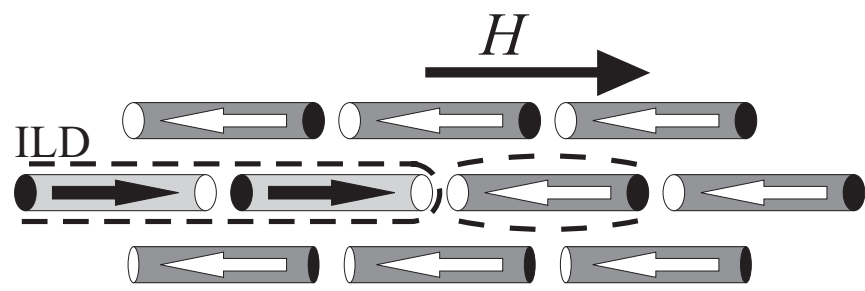

FIG. 5. Schematics of reversal conditions for the least stable bar element, next to the "head" of LD. 
elements in residual noninverted clusters, compare Figs. 3(c) and $(\mathrm{g})]$.

In more general, the geometry dependence of magnetic superlattices can be analyzed considering the above rec-and rho-systems as the extreme cases in the continuous series of superlattices obtained by relative shifts $\delta x$ between neighbor chains of bar elements (along the $x$-axis in Fig. 1). Then it is obvious that the observed and calculated difference $\delta H_{r h o}-$ $\delta H_{r e c}$ is maximum in this series. Also, since the values of $\delta H_{r h o}$ and $\delta H_{\text {rec }}$ have opposite signs, it can be expected that, when varying $\delta x$ from 0 (rec-case) to $(a+b) / 2$ (rho-case), there exists a special value $x_{0}$ that makes the superlattice coercivity to coincide with that by a single bar element (that is, effective cancellation of interbar magnetostatic interactions happens). Otherwise, at increasing the transversal distance between the chains, the interaction effect (as the rec-rho difference) will monotonously decrease.
In summary, the experimental, numerical, and analytical studies on magnetic states of artificial superlattices of magnetic bar elements show unusual collective properties of these systems that, besides the known effects of distance between elements and their aspect ratio, ${ }^{4}$ are also sensitive to the symmetry of superlattice. The found lattice geometry effect on the system coercivity can be controllably enhanced by a proper choice of element and lattice parameters for tailoring advanced performance of artificial magnetic media in technology applications.

The support of this work by Spanish and Portuguese Grants Nos. HP2008-0032, FIS2008-6249, PCTI IB08-106, FCT SFRH/BPD/72329/2010 (J.M.T.) is gratefully acknowledged. G.N.K. acknowledges support from Portuguese FCT through the "Ciencia 2007" program. Work in Ukraine was supported by STCU through the Project No. 5210.
${ }^{1}$ C. A. Ross, Annu. Rev. Mater Res. 31, 203 (2000).

${ }^{2}$ J. I. Martin, J. Nogues, K. Liu, J. L. Vicente, and I. K. Schuller, J. Magn. Magn. Mater. 256, 449 (2003).

${ }^{3}$ J. Mejia-Lopez, P. Soto, and D. Altbir, Phys. Rev. B 71, 104422 (2005).

${ }^{4}$ T. Schneider, A. A. Serga, B. Leven, B. Hillebrands, R. L. Stamps, and M. P. Kostylev, Appl. Phys. Lett. 92, 022505 (2008).

${ }^{5}$ Y. W. Liu, Z. W. Hou, S. Gliga, and R. Hertel, Phys. Rev. B 79, 104435 (2009).

${ }^{6}$ J. D. Medina, L. Piraux, and A. Encinas, Appl. Phys. Lett. 96, 042504 (2010).

${ }^{7}$ P. Krone, D. Makarov, T. Schrefl, and M. Albrecht, Appl. Phys. Lett. 97, 082501 (2010).

${ }^{8}$ A. Vogel, A. Drews, T. Kamionka, M. Bolte, and G. Meier, Phys. Rev. Lett. 105, 037201 (2010).

${ }^{9}$ Y. F. Hou, Q. F. Zhan, and K. M. Krishnan, Appl. Phys. Lett. 98, 042510 (2011).
${ }^{10}$ P. V. Bondarenko, A. Yu. Galkin, B. A. Ivanov, C. E. Zaspel, Phys. Rev. B 81, 224415 (2010).

${ }^{11}$ M. D. Costa, Y. G. Pogorelov, Phys. Stat. Sol. (a) 189, 923 (2002).

${ }^{12}$ A. Imre, G. Csaba, G. H. Bernstein, W. Porod, and V. Metlushko, Superlattices Microstruct. 34, 513 (2003).

${ }^{13}$ V. Novosad, K. Yu. Guslienko, H. Shima, Y. Otani, S. G. Kim, K. Fukamichi, N. Kikuchi, O. Kitakami, and Y. Shimada, Phys. Rev. B 65, R060402 (2002).

${ }^{14}$ G. N. Kakazei, Y. G. Pogorelov, M. D. Costa, T. Mewes, P. E. Wigen, P. C. Hammel, V. O. Golub, T. Okuno, and V. Novosad, Phys. Rev. B 74, 060406 (2006).

${ }^{15}$ A. A. Awad, G. R. Aranda, D. Dieleman, K. Y. Guslienko, G. N. Kakazei, B. A. Ivanov, and F. G. Aliev, Appl. Phys. Lett. 97, 132501 (2010).

${ }^{16}$ R. Morales, J. I. Martin, M. Velez, and J. M. Alameda, Eur. Phys. J. B 40, 463 (2004).

${ }^{17}$ M. J. Donahue and D. G. Porter, OOMMF User's Guide, Version 1.0, Interagency Report NISTIR 6376, NIST, Gaithersburg, 1999. 